\title{
Finger-Knuckle-Print Verification Based On Band-Limited Phase-Only Correlation
}

\author{
Lin Zhang, Lei Zhang, and David Zhang \\ Biometrics Research Center, Department of Computing, \\ The Hong Kong Polytechnic Univeristy \\ \{cslinzhang, cslzhang, csdzhang\}@comp.polyu.edu.hk
}

\begin{abstract}
This paper investigates a new automated personal authentication technique using finger-knuckle-print (FKP) imaging. First, a specific data acquisition device is developed to capture the FKP images. The local convex direction map of the FKP image is then extracted, based on which a coordinate system is defined to align the images and a region of interest (ROI) is cropped for feature extraction and matching. To match two FKPs, we present a BandLimited Phase-Only Correlation (BLPOC) based method to register the images and further to evaluate their similarity. An FKP database is established to examine the performance of the proposed method, and the promising experimental results demonstrated its advantage over the existing finger-back surface based biometric systems.
\end{abstract}

Keywords: Biometrics, finger-knuckle-print, personal authentication

\section{Introduction}

Personal authentication is a common concern to both industries and academic research due to its numerous applications. Biometrics, which refers to the unique physiological or behavioral characteristics of human beings, can be used to distinguish between individuals. In the past three decades, researchers have exhaustively investigated the use of a number of biometric characteristics [1].

Recently, it has been noticed that the texture in the outer finger surface has the potential to do personal authentication. Woodward et al. [2-3] set up a 3-D hand database with the Minolta 900/910 sensor and they extracted 3-D features from finger surface to identify a person's identity. However, they did not provide a practical solution to establishing an efficient system using the outer finger surface features. The cost, size and weight of the Minolta 900/910 sensor limit the use of it in a practical biometric system, and the time-consuming 3-D data acquisition and processing limit its use in real-time applications. Later, Kumar and Ravikanth [4-5] proposed another approach to personal authentication by using 2-D finger-back surface imaging. They developed a device to capture hand-back images and then extracted the finger knuckle areas by some preprocessing steps. The subspace analysis methods such as PCA, LDA and ICA were combined to do feature extraction and matching. With 
Kumar et al.'s design, the acquisition device is doomed to have a large size because nearly the whole hand back area has to be captured, despite the fact that the finger knuckle area only occupies a small portion of the acquired image. Furthermore, subspace analysis methods may be effective for face recognition but they may not be able to effectively extract the distinctive line features from the finger knuckle surface.

This paper presents a novel system for online personal authentication based on finger-knuckle-print (FKP), which refers to the inherent skin pattern of the outer surface around the phalangeal joint of one's finger. A specially designed acquisition device is constructed to collect FKP images. Unlike the systems in [3] and [5] which first capture the image of the whole hand and then extract the finger or finger knuckle surface areas, the proposed system captures the image around the finger knuckle area of a finger directly, which largely simplifies the following data preprocessing steps. Meanwhile, with such a design the size of the imaging system can be greatly reduced, which improves much its applicability. Since the finger knuckle will be slightly bent when being imaged in the proposed system, the inherent finger knuckle print patterns can be clearly captured and hence the unique features of FKP can be better exploited. For matching FKPs, we present an efficient and effective Band-Limited Phase-Only Correlation based method. Compared with the existing finger knuckle surface based biometric systems [2-5], the proposed system performs much better in terms of both recognition accuracy and speed.

The rest of this paper is organized as follows. Section 2 introduces the design and structure of the FKP image acquisition device. Section 3 describes the FKP image preprocessing and ROI extraction methods. Section 4 investigates the BLPOC-based FKP matching. Section 5 reports the experimental results. Finally, conclusions are presented in section 6 .

\section{The FKP Recognition System}

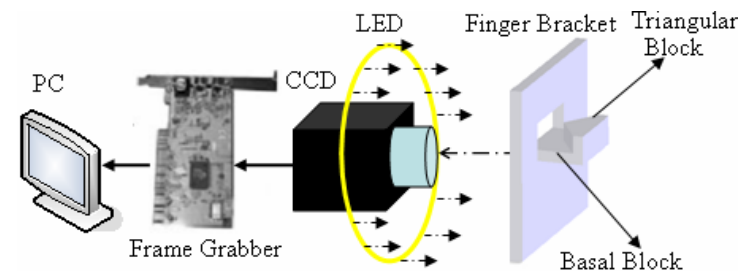

(a)

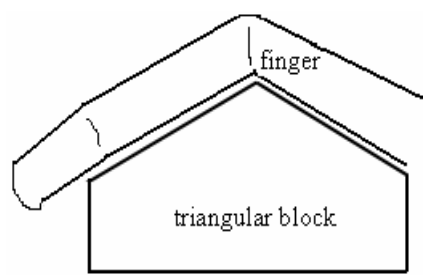

(b)

Fig. 1: FKP image acquisition device.

The proposed FKP recognition system is composed of an FKP image acquisition device and a data processing module. The device (referring to Fig. 1-a) is composed of a finger bracket, a ring LED light source, a lens, a CCD camera and a frame grabber. The captured FKP image is inputted to the data processing module, which comprises three basic steps: ROI (region of interest) extraction, feature extraction, and feature matching. Refer to Fig. 1-a, a basal block and a triangular block are used to fix the position of the finger joint. The vertical view of the triangular block is illustrated in Fig. 1-b. Fig. 2-a shows a sample image acquired by the developed device. 


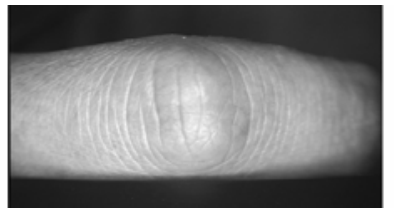

(a)

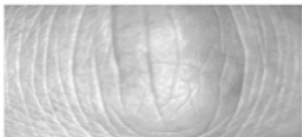

(b)

Fig. 2: (a) is a sample FKP image; (b) is the ROI image of (a).

\section{ROI Extraction}

It is necessary to construct a local coordinate system for each FKP image. With such a coordinate system, an ROI can be cropped from the original image for reliable feature extraction. The detailed steps for setting up such a coordinate system are as follows.

Step 1: determine the $X$-axis of the coordinate system. The bottom boundary of the finger can be easily extracted by a Canny edge detector. Actually, this bottom boundary is nearly consistent to all FKP images because all the fingers are put flatly on the basal block in data acquisition. By fitting this boundary as a straight line, the $X$-axis of the local coordinate system is determined.

Step 2: crop a sub-image $I_{S}$. The left and right boundaries of $I_{S}$ are two fixed values evaluated empirically. The top and bottom boundaries are estimated according to the boundary of real fingers and they can be obtained by a Canny edge detector.

Step 3: Canny edge detection. Apply the Canny edge detector to $I_{S}$ to obtain the edge map $I_{E}$.

Step 4: convex direction coding for $I_{E}$. We define an ideal model for FKP "curves". In this model, an FKP "curve" is either convex leftward or convex rightward. We code the pixels on convex leftward curves as "1", pixels on convex rightward curves as " -1 ", and the other pixels not on any curves as "0".

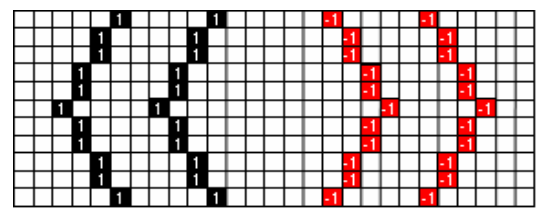

Fig. 3: Illustration for convex direction coding scheme.

Fig. 3 illustrates this convex direction coding scheme and the pseudo codes are presented as follows:

\section{Convex_Direction_Coding $\left(I_{E}\right)$}

Output: $I_{C D}$ (convex direction code map)

$y_{\text {mid }}=$ height of $I_{E} / 2$;

for each $I_{E}(i, j)$ : 


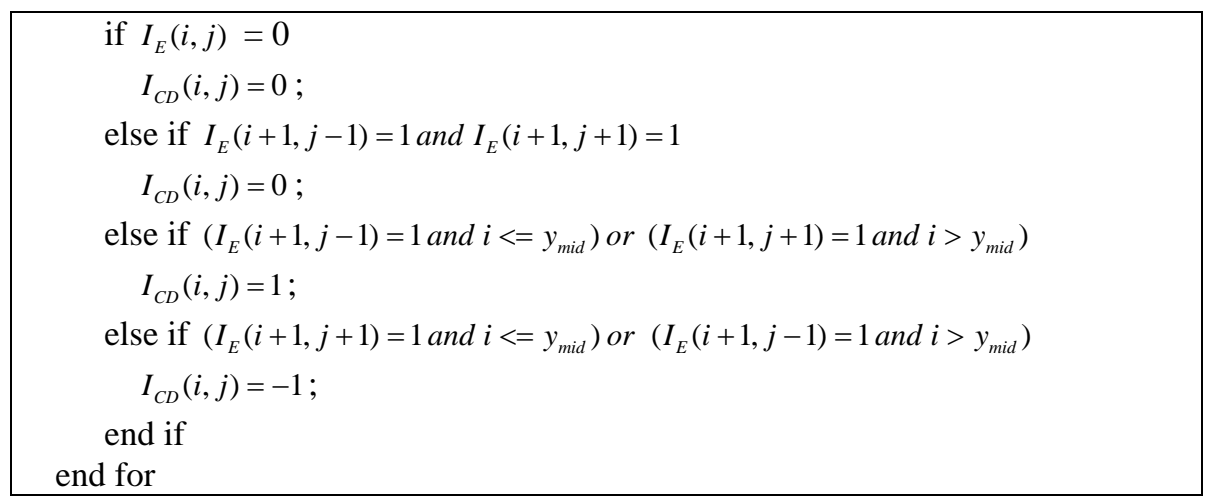

Step 5: determine the $Y$-axis of the coordinate system. For an FKP image, “curves" on the left part of phalangeal joint are mostly convex leftward and those on the right part are mostly convex rightward. Meanwhile, "curves" in a small area around the phalangeal joint do not have obvious convex directions. Based on this observation, at a horizontal position $x$ ( $x$ represents the column) of an FKP image, we define the “convexity magnitude” as:

$$
\operatorname{conMag}(x)=a b s\left(\sum_{w} I_{C D}\right)
$$

where $W$ is a window being symmetrical about the axis $X=x . W$ is of the size $d \times h$, where $h$ is the height of $I_{S}$. The characteristic of the FKP image suggests that con$\operatorname{Mag}(x)$ will reach a minimum around the center of the phalangeal joint and this position can be used to set the $Y$-axis of the coordinate system. Let

$$
x_{0}^{\prime}=\arg \min (\operatorname{conMag}(x))
$$

Then $X=x_{0}^{\prime}$ is set as the $Y$-axis.

Step 6: crop the ROI image. Now that we have fixed the $X$-axis and $Y$-axis, the local coordinate system can then be determined and the ROI sub-image $I_{R O I}$ can be extracted with a fixed size. Fig. 2-b shows an example of the extracted ROI images.

\section{BLPOC-Based FKP Matching}

Given two FKP ROIs, a matching algorithm determines their degree of similarity. A BLPOC based FKP matching algorithm is presented in this section.

\subsection{Phase-Only Correlation (POC)}

Phase-Only Correlation (POC) has been widely used for image registration tasks [68], and recently it has been adopted in some biometric systems as a similarity measure [9-11]. POC based method relies on the translation property of the Fourier transform. Let $f$ and $g$ be the two images that differ only by a displacement $\left(x_{0}, y_{0}\right)$ i.e.

$$
g(x, y)=f\left(x-x_{0}, y-y_{0}\right)
$$


Their corresponding Fourier transforms $G(u, v)$ and $F(u, v)$ will be related by

$$
G(u, v)=e^{-j 2 \pi\left(u x_{0}+v y_{0}\right)} F(u, v)
$$

The cross-phase spectrum $R_{G F}(u, v)$ between $G(u, v)$ and $F(u, v)$ is given by

$$
R_{G F}(u, v)=\frac{G(u, v) F^{*}(u, v)}{\left|G(u, v) F^{*}(u, v)\right|}=e^{-j 2 \pi\left(u x_{0}+v y_{0}\right)}
$$

where $F^{*}$ is the complex conjugate of $F$. By taking inverse Fourier transform of $R_{G F}$ back to the time domain, we will have a Dirac impulse centered on $\left(x_{0}, y_{0}\right)$.

In practice, we should consider the finite discrete representations. Consider two $M \times N$ images, $f(m, n)$ and $g(m, n)$, where the index ranges are $m=-M_{0}, \ldots, M_{0}\left(M_{0}>0\right)$ and $n=-N_{0}, \ldots, N_{0}\left(N_{0}>0\right)$, and $M=2 M_{0}+1$ and $N=2 N_{0}+1$. Denote by $F(u, v)$ and $G(u, v)$ the 2D DFTs of the two images and they are given by

$$
\begin{aligned}
& F(u, v)=\sum_{m=-M_{0}}^{M_{0}} \sum_{n=-N_{0}}^{N_{0}} f(m, n) e^{-j 2 \pi\left(\frac{m u}{M}+\frac{n v}{N}\right)}=A_{F}(u, v) e^{j \phi_{F}(u, v)} \\
& G(u, v)=\sum_{m=-M_{0}}^{M_{0}} \sum_{n=-N_{0}}^{N_{0}} g(m, n) e^{-j 2 \pi\left(\frac{m u}{M}+\frac{n v}{N}\right)}=A_{G}(u, v) e^{j \phi_{G}(u, v)}
\end{aligned}
$$

where $u=-M_{0}, \ldots, M_{0}, v=-N_{0}, \ldots, N_{0}, A_{F}(u, v)$ and $A_{G}(u, v)$ are amplitude components, and $\phi_{F}(u, v)$ and $\phi_{G}(u, v)$ are phase components. Then, the cross-phase spectrum $R_{G F}(u, v)$ between $G(u, v)$ and $F(u, v)$ is given by

$$
R_{G F}(u, v)=\frac{G(u, v) F^{*}(u, v)}{\left|G(u, v) F^{*}(u, v)\right|}=e^{j\left\{\phi_{G}(u, v)-\phi_{F}(u, v)\right\}}
$$

The POC function $p_{g f}(m, n)$ is the 2D Inverse DFT (IDFT) of $R_{G F}(u, v)$ :

$$
p_{g f}(m, n)=\frac{1}{M N} \sum_{u=-M_{0}}^{M_{0}} \sum_{v=-N_{0}}^{N_{0}} R_{G F}(u, v) e^{j 2 \pi\left(\frac{m u}{M}+\frac{n v}{N}\right)}
$$

If the two images $f$ and $g$ are similar, their POC function will give a distinct sharp peak. On the contrary, if they are not similar, the peak value will drop significantly. Thus, the height of the peak value can be used as a similarity measure, and the location of the peak shows the translational displacement between the two images.

\subsection{Band-Limited Phase-Only Correlation (BLPOC)}

In the POC-based image matching method, all the frequency components are involved. However, high frequency tends to emphasize detail information and can be prone to noise. To eliminate meaningless high frequency components, K. Ito et al. [9] proposed the Band-Limited Phase-Only Correlation (BLPOC).

The BLPOC limits the range of the spectrum of the given FKP image. Assume that the ranges of the inherent frequency band of FKP texture are given by $u=-U_{0}, \ldots, U_{0}$ and $v=-V_{0}, \ldots V_{0}$, where $0<=U_{0}<=M_{0}, 0<=V_{0}<=N_{0}$. Thus, the effective size of spectrum is given by $L_{1}=2 U_{0}+1$ and $L_{2}=2 V_{0}+1$. The BLPOC function is defined as

$$
p_{g f}^{U_{0} V_{0}}(m, n)=\frac{1}{L_{1} L_{2}} \sum_{u=-U_{0}}^{U_{0}} \sum_{v=-V_{0}}^{V_{0}} R_{G F}(u, v) e^{j 2 \pi\left(\frac{m u}{L_{1}}+\frac{n v}{L_{2}}\right)}
$$


where $m=-U_{0}, \ldots, U_{0}$ and $n=-V_{0}, \ldots, V_{0}$. When two images are similar, their BLPOC function gives a distinct sharp peak. Also, the translational displacement between the two images can be estimated by the correlation peak position. Experiments indicate that the BLPOC function provides a much higher discrimination capability than the original POC function.

\subsection{BLPOC-Based FKP Matching}

Given two FKP ROIs $f(m, n)$ and $g(m, n)$, we assume that there is only translational displacement between them. Thus, the BLPOC-based FKP matching process is quite straightforward and is summarized as follows:

\section{FKP_Matching $(f(m, n), g(m, n))$}

Output: matching_score

register $f$ and $g$ based on BLPOC method;

extract overlapping areas $f^{\prime}$ and $g$ from registered $f$ and $g$, respectively;

if area $\left(f^{\prime}\right) / \operatorname{area}(f)<$ threshold

$$
P=\operatorname{BLPOC}(f, g)
$$

else

$$
P=\operatorname{BLPOC}\left(f^{\prime}, g^{\prime}\right)
$$

end

matching_score $=\max (P)$;

\section{Experimental Results}

Database establishment. An FKP database was established. The FKP images were collected from 165 volunteers, including 125 males and 40 females. Among them, 143 subjects are 20 30 years old and the others are 30 50 years old. We collected the images in two separate sessions. In each session, the subject was asked to provide 6 images for each of the left index finger, the left middle finger, the right index finger and the right middle finger. Therefore, 48 images from 4 fingers were collected from each subject. In total, the database contains 7,920 images from 660 different fingers.

Experiment 1. In the first experiment, we took images captured in the first session as the gallery set and images captured in the second session as the probe set. Therefore, there were 660 classes and 3,960 images in the gallery set and the probe set each. Each image in the probe set was matched against the all the images in the gallery set. A match was counted as genuine if the two FKPs were from the same finger; otherwise the match was counted as imposter. As a result, the numbers of genuine matches and imposter matches were 23,760 and 7,828,920, respectively. The EER (Equal Error Rate) wet got was $1.68 \%$, which was quite promising. Fig. 4a depicts the corresponding FAR (False Acceptance Rate) and FRR (False Rejection Rate) curves. The distance distributions of genuine matching and imposter matching obtained in this 
experiment are plotted in Fig. 4b. Experimental results indicate that the proposed system has a good capability to verify a person's identity.

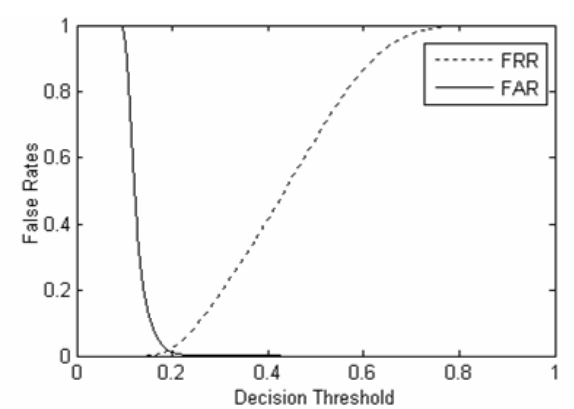

(a)

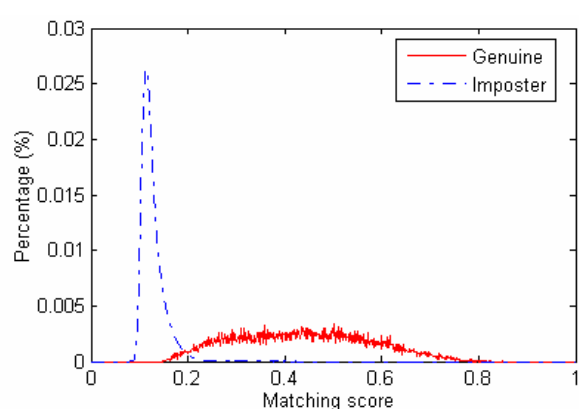

(b)

Fig. 4: (a) FAR and FRR curves obtained in experiment 1. (b) Distance distributions of genuine matching and imposter matching obtained in experiment 1.

Experiment 2. The goal of this experiment was to investigate the system's performance when we fuse information from 2 or more fingers of a person. In fact, at such a case the system works as a kind of multi-modal system with a single biometric trait but multiple units. We adopt the SUM fusing rule as follows:

$$
s_{\text {sum }}=\sum s_{i}
$$

where $s_{i}$ is the matching score of the client's $i_{t h}$ finger.

Table 1. Experimental results in experiment 2

\begin{tabular}{|l|l|l|l|l|l|l|}
\hline Method & $\begin{array}{l}\text { gallery } \\
\text { classes }\end{array}$ & $\begin{array}{l}\text { gallery } \\
\text { samples }\end{array}$ & $\begin{array}{l}\text { probe } \\
\text { classes }\end{array}$ & $\begin{array}{l}\text { probe } \\
\text { samples }\end{array}$ & Finger types for fusion & $\begin{array}{l}\text { EER } \\
(\%)\end{array}$ \\
\hline Ours & 165 & 990 & 165 & 990 & l-index, l-middle & 0.72 \\
\hline Ours & 165 & 990 & 165 & 990 & r-index, r-middle & 0.31 \\
\hline Ours & 165 & 990 & 165 & 990 & l-index, r-index & 0.40 \\
\hline Ours & 165 & 990 & 165 & 990 & l-middle, r-middle & 0.31 \\
\hline Woodward [3] & 132 & 660 & 177 & 531 & r-index, r-middle, r-ring & 5.50 \\
\hline Kumar [5] & 105 & 420 & 105 & 210 & index, middle, ring, little & 1.39 \\
\hline
\end{tabular}

We tested several different fusions of fingers and the results are presented in Table 1 , from which it can be easily observed that by integrating information from more fingers the recognition performance of the system could be largely improved. We also present the results extracted from [3] and [5] in Table 1 for comparison. It is clearly shown that the proposed system performed much better even though we incorporated information from fewer fingers.

Speed. The software was implemented with Visual C\#.Net 2005 on a Dell Inspiron 530s PC embedded Intel E6500 process and 2G RAM. The execution time for data preprocessing and ROI extraction was $216 \mathrm{~ms}$. The time for one BLPOC-based matching was $4.2 \mathrm{~ms}$. Thus, the total execution time for one verification operation was less than $0.3 \mathrm{~s}$ in our prototype system, which was fast enough for real-time applications. 


\section{Conclusions}

This paper presented a new approach to online personal authentication using fingerknuckle-print (FKP), which has distinctive line features. A cost-effective FKP system, including a novel image acquisition device and the associated data processing algorithms, was developed. To efficiently match the FKPs, we proposed a BLPOC based FKP matching method. Extensive experiments demonstrated the efficiency and effectiveness of the proposed technique. Compared with other existing finger back surface based systems, the proposed FKP authentication has merits of high accuracy, high speed, small size and cost-effective.

\section{Acknowledgments}

The work is partially supported by the Edward Sai Kim Hotung Fund (5-ZH52), the HK-PolyU Internal Competitive Research Grant (G-YH54), and the Hong Kong RGC General Research Fund (PolyU 5351/08E).

\section{References}

1. Jain, A.K., Flynn, P., Ross, A. (ed.): Handbook of Biometrics. Springer, (2007)

2. Woodard, D.L., Flynn, P.J.: Personal identification utilizing finger surface features. In: Proc. CVPR, vol. 2, pp. 1030-1036 (2005)

3. Woodard, D.L., Flynn, P.J.: Finger surface as a biometric identifier. Computer Vision and Image Understanding 100 (3), 357-384 (2005)

4. Ravikanth, C., Kumar, A.: Biometric authentication using finger-back surface. In: Proc. CVPR, pp. 1-6 (2007)

5. Kumar, A., Ravikanth, C.: Personal authentication using finger knuckle surface. IEEE Trans. Information Forensics and Security 4 (1), 98-109 (2009)

6. Kuglin, C.D., Hines, D.C.: The phase correlation image alignment method. In: Proc. Int. Conf. on Cybernetics and Society, pp. 163-165 (1975)

7. Chen, Q.S., Defrise, M., Deconinck, F.: Symmetric phase-only matched filtering of FourierMellin transforms for image registration and recognition. IEEE Trans. PAMI 16 (12), 11561168 (1994)

8. Srinivasa Reddy, B., Chatterji, B.N.: An FFT-based technique for translation, rotation, and scale-invariant image registration. IEEE Trans. IP 5 (8), 1266-1271 (1996)

9. Ito, K., Nakajima, H., Kobayashi, K., Aoki, T., Higuchi, T.: A fingerprint matching algorithm using phase-only correlation. IEICE Trans. Fundamentals E87-A (3), 682-691 (2004)

10. Zhang, J.X., Ou, Z.Y., Wei, H.L.: Fingerprint matching using phase-only correlation and Fourier-Mellin transforms. In: Proc. Int. Conf. ISDA, vol. 2, pp. 379-383 (2006)

11. Miyazawa, K., Ito, K., Aoki, T., Kobayashi, K., Nakajima, H.: An effective approach for iris recognition using phase-based image matching. IEEE Trans. PAMI 20 (10), 1741-1756 (2008) 